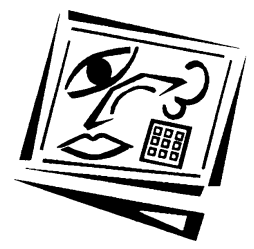

\title{
Mathematics learning forum: Role of ICT in the construction of pre-service teachers' content knowledge schema
}

\author{
Mohan Chinnappan \\ University of Wollongong
}

\begin{abstract}
Recent interest in the topic of knowledge bases for teachers of mathematics has led to a sustained analysis of how teacher knowledge influences interactions with their students. The results of this body of research have focused on identifying and describing the growth of mathematics teachers' content and pedagogical content knowledge. In particular, it has been argued that there is a need to examine how mathematics teachers who are new to the classroom construct and structure their knowledge base (National Council of Teachers of Mathematics, 2000). Here I examine this issue by exploring the role of an online mathematics learning forum in creating an environment within which a group of prospective teachers exchanged views about a topic on arithmetic. The online forum was established via WebCT. The results suggest that WebCT provided a convenient and non-threatening medium in which to generate descriptions about mathematics teachers' knowledge and understanding.
\end{abstract}

\section{Introduction}

Current models of mathematics instruction are based on the assumption that mathematical knowledge and understandings are unlikely to be discovered by children through their own empirical enquiry, but instead are constructed when they engage socially in talk and action about shared problems and tasks. While there is a general consensus on the need to facilitate dialogue among learners and teachers of mathematics, less is known about the design and role of learning environments that would impact positively on developing a deeper appreciation of the nature of mathematics, what it means 'to do' mathematics and to teach mathematics.

In this study I examine this issue by exploring the role of an online mathematics learning forum in creating a rich learning environment 
within which a group of pre-service teachers exchanged views about a topic in arithmetic. The online forum was established via WebCT.

\section{Online mathematics learning forums}

Current models of mathematics teacher education place emphasis on facilitating student teachers' learning by establishing a community of learners, where lectures and tutorials support active exploration of learning theories and best practices in teaching. While tutorials and lectures provide important avenues to engage student teachers, the quality of this interaction is limited. Because student teachers spend a considerable amount of their time pursuing other subjects and interact with children via practicum, there are fewer opportunities for them to dialogue with their peers. Thus there is a need to find a medium that would help establish a network for collaboration and group work that is not constrained by time or physical presence.

In recent years online learning has received considerable attention and support from the mathematics teaching community, including teacher educators. The current trend is to go beyond using online facilities for teaching, and employ this as a powerful tool in creating a forum for encouraging learning by sharing ideas with colleagues. Student teachers could now share views about innovative teaching techniques for K-6 mathematics, as well as communicate with their lecturers about children's learning styles and the development of appropriate assessment techniques.

There are a number of practical and pedagogical advantages associated with building virtual learning communities for student teachers. On the pragmatic end is the convenience of information and communication technologies (ICT). Because student teachers spend much of their study time in formal classes, there is insufficient time available for exchanges with fellow students and practicing professionals. Online affords student teachers the option of communicating with peers at times that are convenient for them. Prospective teachers are able to establish a routine for the exchange of ideas that adjusts to the demands of other tertiary subjects and commitments.

During online discussions, members of a group may exchange ideas, share concerns, reflect on teaching strategies and learn from one another as they converse with colleagues. The exploration of mathematical concepts and teaching with a community of peers could support the development of new understandings about the concept in question, and examine potential strategies for supporting children who experience learning difficulties. 


\section{Theoretical considerations}

\section{Conceptual framework}

In order to analyse prospective teachers' understanding of teaching mathematical concepts, I consider ways these students might think about children's prior knowledge and experiences, and how this knowledge could interact with learning activities designed by the teacher. Recent research, particularly about the development of expertise in mathematics teaching, indicates that there are three major components relating to the knowledge base of teachers and enabling them to perform their role effectively. These are:

1. teachers' mathematical content knowledge,

2. the organisation of this knowledge, and

3. the blend of knowledge of content and pedagogy.

Mathematical content knowledge includes information such as mathematical concepts, rules and associated procedures for problem solving. The organisation of the content knowledge refers to the links that teachers construct between the various components of content knowledge. The blend of content and pedagogical knowledge includes understanding why some children experience difficulties when learning a particular concept, while others find it easy to assimilate, knowledge about useful ways to conceptualise and represent a chosen concept (Feiman-Nemser, 1990), the quality of explanations that teachers generate prior to and during instruction (Leinhardt, 1987), and characteristics of the learners. This latter knowledge has also been labeled as pedagogical content knowledge (Shulman, 1986).

In recent years researchers interested in improving children's mathematical performance have argued that the quality of a teacher's knowledge has a strong influence on how that knowledge is accessed and exploited during planning for a lesson and instruction (Clark and Peterson, 1986; Lawson and Chinnappan, 1994; Schoenfeld, 1992). Critically, teacher knowledge and planning can be argued to influence and be influenced by children's reactions to learning activities. Thus, I work from the emergent perspective (Cobb, 1994; Simon, 1995) where children's activity and mathematical understandings are assumed to evolve as they work within social groups.

In this study I apply the constructivist framework for the analysis of preservice teachers' ${ }^{\prime}$ knowledge of multiplication and the teaching of multiplication. According to this framework, one aspect of student teachers' professional development can be interpreted as involving construction of links between mathematical concepts and the teaching of 
these concepts. A major assumption in this analysis is that an individual student teacher's quality of understanding is based on the nature of the connections that he or she is able to build between his or her prior knowledge, and the learning experiences that are provided by teacher education courses and practicum.

\section{Modelling, learning and mathematics teaching}

Modelling involves the establishment of links among representations of a mathematical concept and its relationship to other concepts. More importantly, a model needs to externalise the links to the learner in ways that would help him or her visualise them (English and Halford, 1995). Consider the concept of symmetry in the study of geometry of shapes. There are a number of ways to categorise symmetry, such as reflection and rotation. When children are introduced to the concept of symmetry, they could begin by recognising the properties and creating shapes that symbolise symmetry. As their understanding matures, their representation of symmetry would include a network of nodes and relations that involve reflection, rotation and related concepts. This network of items of information forms a schema for symmetry (Chinnappan, 1998; Marshall, 1995). Such a schema may also have information about applications of symmetry and rules or procedures about using symmetry in the solution of problems. Thus modelling involves the depiction of the relations that are embedded in a schema both graphically or concretely.

Having constructed a model for a concept, teachers could go further and consider exploration of that model. Model exploration could involve activities that help children gain insight into the many interwoven connections that may have been established among the relevant knowledge components of the model. Such an exploration could reveal the structure of schemas to the learner. In this way children can be expected to access higher levels of prior knowledge and attempt to integrate that knowledge with elements of the model that is being constructed. The modelling process could also contribute to the expansion of networks of schemas that are associated with mathematical concepts resulting in deeper understandings.

Thus, model exploration activities can be expected to extend links that have already been built, and help children identify the various representations that are anchored by the model such as concepts, relations, patterns and translations. Modelling activities must also have an inbuilt flexibility to help children externalise constituents of a model. These activities need to be grounded within the experiences of children, including observation of concepts in real life contexts. Exploration would 
also reveal children's ability to use that model to conjecture about other situations and solve problems.

\section{Modelling and the development of multiplicative structures}

The above analysis of models and the processes underlying modelling has direct implications for the elucidation of knowledge that underlies children's understanding of multiplication. The notion of model suggests that the understanding of multiplication and its applications is based on the quality of multiplicative structures or schemas stored in long term memory. The teaching of multiplication must therefore assist children to explore different models of multiplication, as a means to building and expanding useful multiplicative structures (Vergnaud, 1988). This outcome could be achieved by adopting an instructional strategy in which teachers employ available resources to model multiplication in different ways. The complex nature of multiplication is reflected in the number of different models that learners can construct with the sub-concepts.

Two models of multiplication are repeated addition and area/rectangular array. These macro models are built on sub-models which in turn are built on schemas of multiples and factors, grouping, properties of multiplication (commutative, associative, distributive) and multiplication algorithms. Repeated addition shows, for example, that $7 \times 5$ is equivalent to $7+7+7+7+7$. It is important for children to understand the relationship between addition and multiplication. That is, multiplying 7 by 5 is equivalent to adding seven fives together. Modelling should aim to help children discover that adding seven fives together will give them the same result as adding five sevens (commutativity). The use of rectangular arrays provides an alternative way to help children visualise multiplication, but this strategy should be grounded in symbol manipulation as well, i.e. writing out $7+7+7+7+7$ and seeing that it is equal to 35 . Equally, children should be able to establish links between the symbols and elements that are located in the cells of the array.

An important conceptual structure underlying these cognitions is counting in multiples. Children must be able to coordinate two composite units in the context of multiplication. For example, in a task involving multiplication of $6 \times 3$, children must visualise six groups of three. The understanding of place value is also a key requirement in performing multiplication operations involving whole numbers, as children ought to recognise that the product is always larger than any of the factors. 


\section{Pedagogical schema for multiplication}

The above analyses of the concept of multiplication in terms of models demonstrate that this is indeed a complex area of K-6 mathematics, and that learning experiences that teachers utilise in the classroom ought to provide opportunities for children to essay the many facets of the concept. The construction of such models is influenced by a number of factors including teachers' pedagogical content knowledge. Shulman's (1986) notion of pedagogical content knowledge posits that teachers need to integrate their own knowledge of mathematics with understandings about the nature of learning and the learner, in order to design effective learning environments. Figure 1 shows a schema that illustrates the type of integration that is relevant to teaching of the concept of multiplication. In this schema for teachers' knowledge there are four principal components, all of which impact on each other. In the current study, this framework is utilised for the analysis of pre-service teachers' knowledge base for teaching.

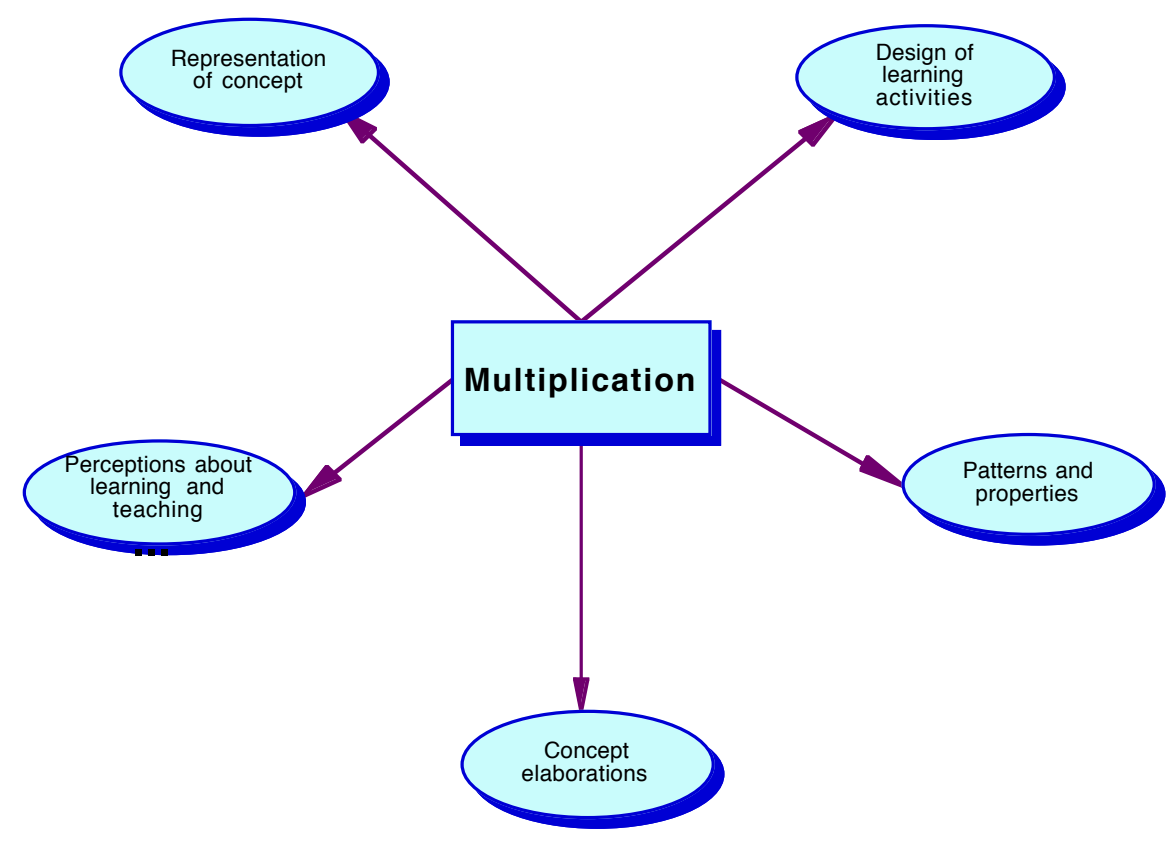

Figure 1: Multiplication schema 


\section{Method}

\section{Participants}

The participants in the present study were 30 pre-service teachers who were enrolled in the third year of their BEd (Primary) program. Prior to the study, these student teachers completed two mathematics methods subjects, both emphasising constructivist principles in primary and early childhood mathematics teaching and learning. Before this study, they completed six weeks of teaching practice. During the two years prior to the present study, the participants completed mathematics discipline requirements for the BEd (Primary) degree, which included number, geometry and algebra. All participants had used computers during their courses, and exhibited high levels of facility with the use of WebCT on the Internet.

\section{Material and procedure}

A hypothetical mathematics lesson plan was developed that would provide a starting point for the online discussion. The lesson focused on the teaching of the concept of multiplication to K-6 children. In this particular lesson plan, the teacher's aim was to use concrete objects to portray multiplication of whole numbers. During the first meeting, all participating student teachers were given an opportunity to analyse the lesson plan and raise questions. Following this meeting, the participants were divided into four groups, and each group was asked to engage in discussions on WebCT for a period of 13 weeks. All communications within each group were recorded in WebCT. At the end of week 13, these comments were downloaded, collated and analysed.

The transcripts of each group's discussions were examined for instances of pedagogically meaningful comments, arguments and suggestions. Elements in each of the three categories referred to participants' contributions that increased the likelihood of enhancing children's understanding of multiplication and applications of multiplications. Comments are parts of student teachers' dialogue that involved opening statements about some aspect of the lesson plan and how that lesson plan could be implemented. Arguments refer to instances where student teachers drew on group members' comments, and developed different points of view about an issue. The category of suggestions indicated that the participants were actually proposing potentially useful ideas about multiplication and classroom strategies to help children grasp the many aspects of multiplication. 
The above categories of exchanges were used in the construction of a series of concept maps. It has been argued that concept maps are powerful tools for the study of spread and structure of concepts and understandings (Jonassen, Beissner and Yacci, 1993). In searching the transcripts, the focus was to identify comments that were relevant to pedagogical content (multiplication) knowledge schemas and consistent with the aforementioned three categories of input. The resulting concept maps for groups 1, 2, 3 and 4 are presented in Figures 2, 3, 4 and 5 respectively. In the construction of the maps, the main concern was to illustrate the nodes, sub-nodes and their relations that provided insight into the modeling of multiplication and teaching. Thus the structure of the maps focused on the nodes and links in a way that would highlight the schema that was emerging during the course of the discussions. Consequently, any statement on the line linking two nodes was not included. The number on each of the nodes does not indicate the order in which that item of knowledge was activated.

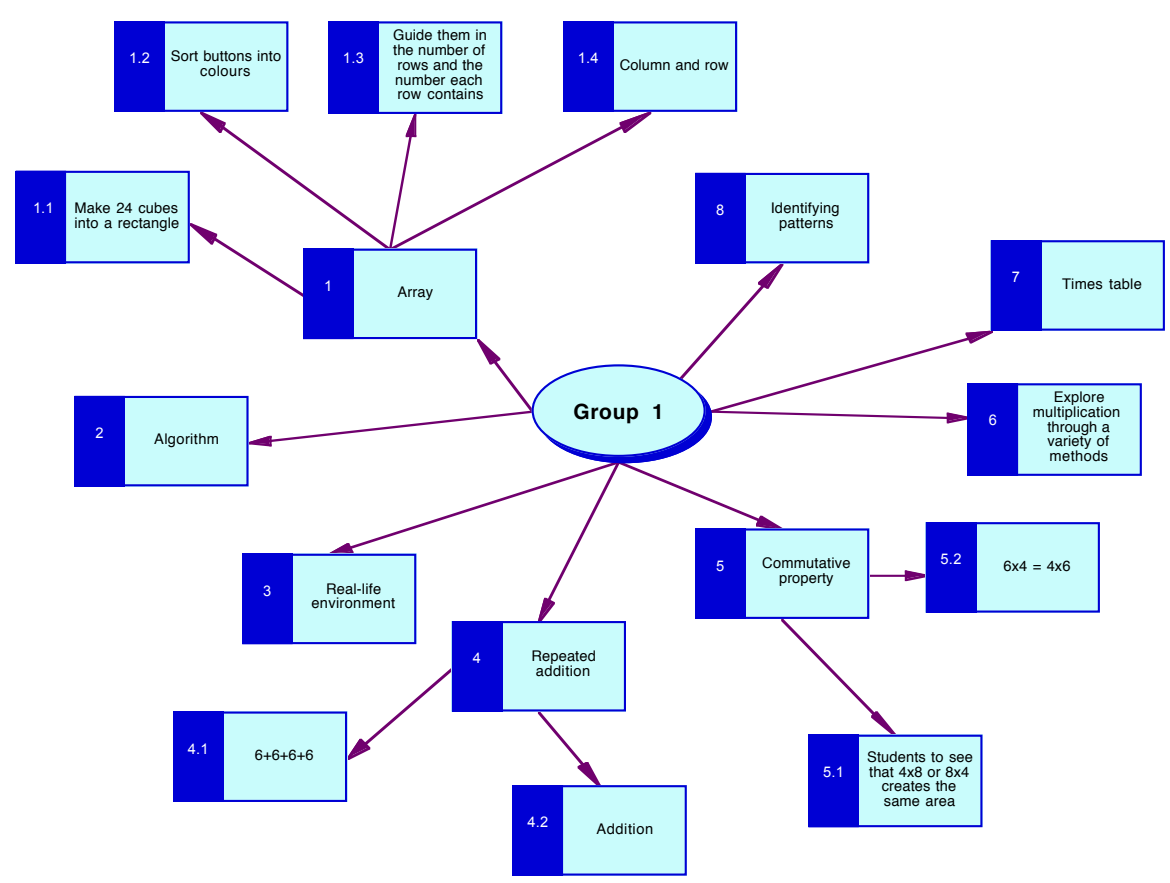

Figure 2: Concept map for Group 1 discussion 


\section{Results}

Figure 2 shows that members of Group 1 had examined the concept of multiplication at some length. The student teachers activated eight key features of multiplication. The modelling of multiplication through the use of arrays generated a high level of interest. These comments are indicated in Figure 2 by Node 1 and the associated subsidiary nodes. The discussion seemed to focus on the arrangement of objects in rows and columns. The comment about the need to look for patterns in an array was a critical step in using arrays to show multiplicative properties, but this connection was not explored in the context of use of arrays. Participants did allude to identification of patterns that were embedded in multiplication operations (Node 8), but in other situations.

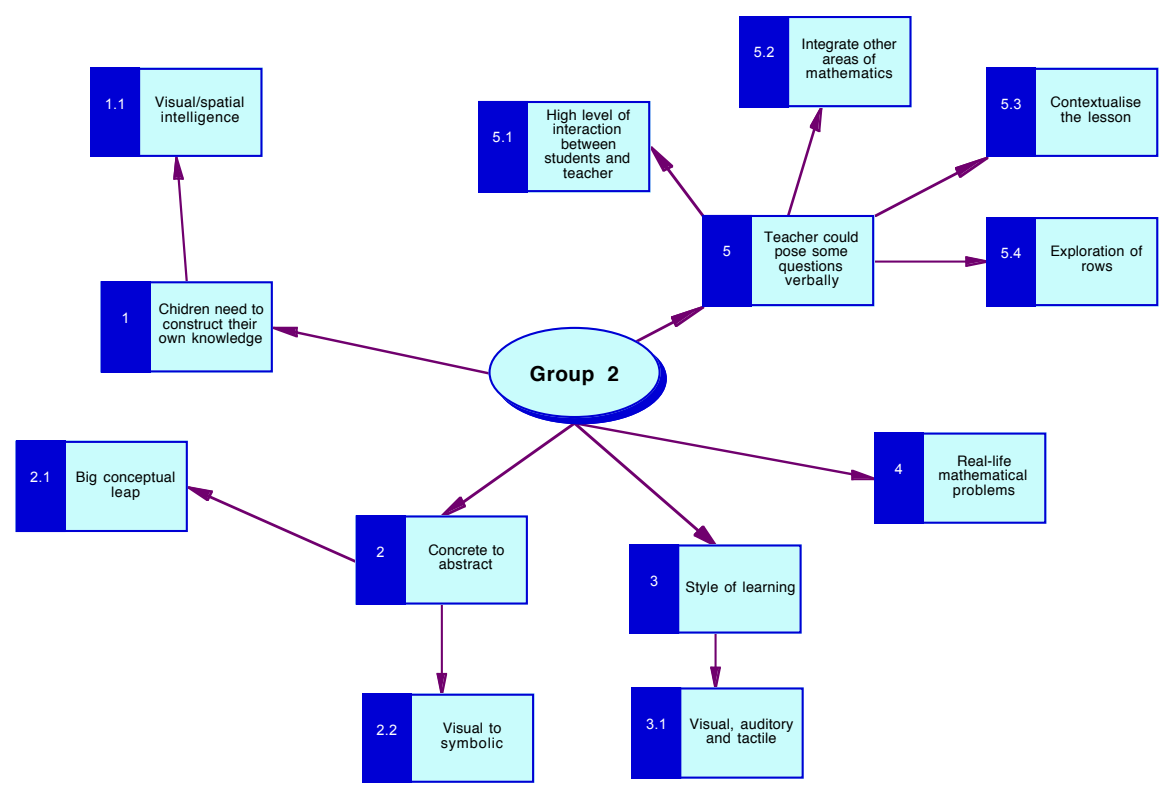

Figure 3: Concept map for Group 2 discussion

Discussions by student teachers from Group 2 (Figure 3) seemed to have centered more on teaching approaches and children's construction of understandings about multiplication. A total of five themes were identified. Posing questions to children seemed to have given rise to further dialogue about how such an approach could encourage children to contextualise the concept of multiplication in the lessons (Subsidiary node 5.3). Interest in the construction of individual knowledge motivated the 
members of Group 2 to explore the role of visual and spatial intelligence in understanding multiplication (Subsidiary node 1.1).

The exchange of ideas in Group 3 (Figure 4) resembled that of Group 2 participants, in that most of the comments posted on WebCT examined the design of learning environments for their children. Node 4 shows that student teachers were interested in providing appropriate concrete material in order to model multiplication operation and its properties. However, the mapping of concepts with corresponding materials was not examined in the exchanges. Node 2 indicates that student teachers were exploring the commutative property of multiplication.

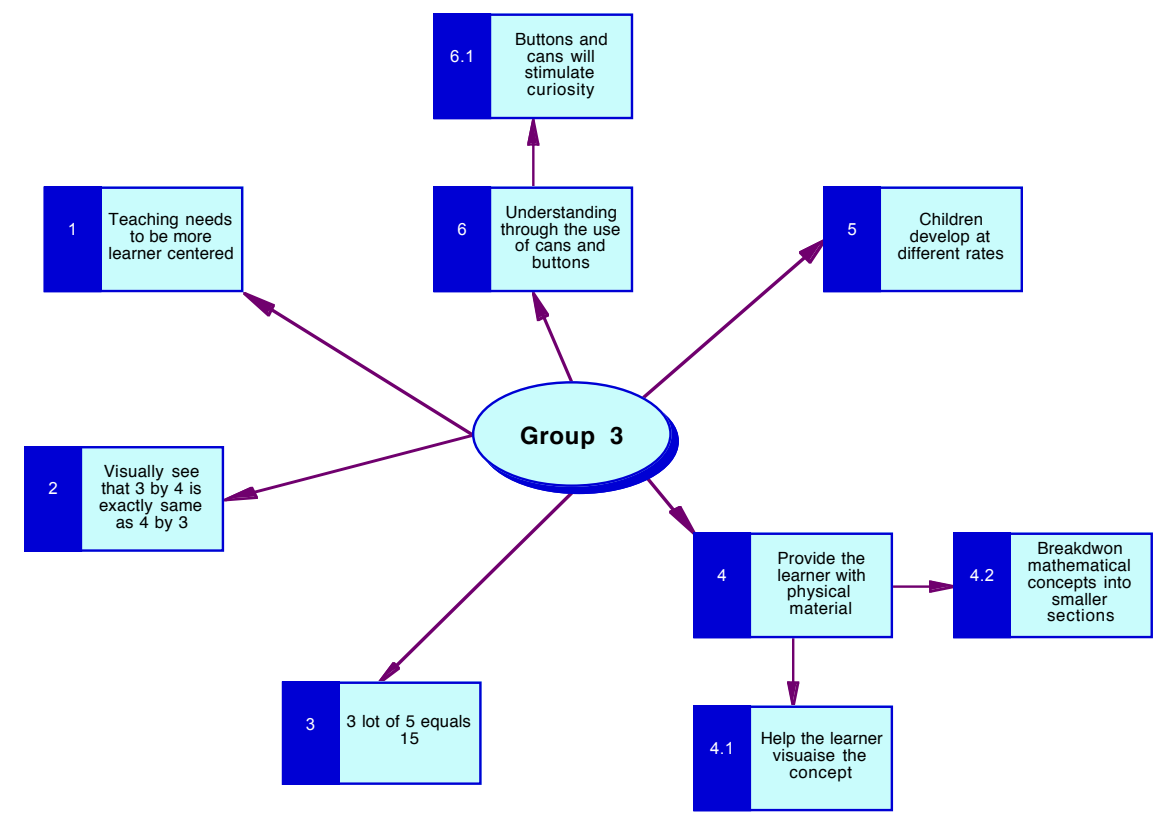

Figure 4: Concept map for Group 3 discussion

Group 4 interactions indicate that student teachers' discussions resulted in longer chains of reasoning about multiplication and the teaching of multiplication than the other groups. This is evidenced by the fact that four out of the six nodes in Figure 5 had subsidiary nodes. The complexity of the concept of multiplication was examined with participants arguing that there was a need to break down the concept (Subsidiary node 1.1). This strategy of decomposing the concept also prompted arguments about the need to consider multiplication as a repeated addition (Subsidiary nodes $1.2,1.21)$. Participants in this group also considered the advantage 
of introducing the concept by different methods. Node 6 shows that participants were concerned about the development of meta-cognitive skills such as checking once a model of multiplication had been constructed.

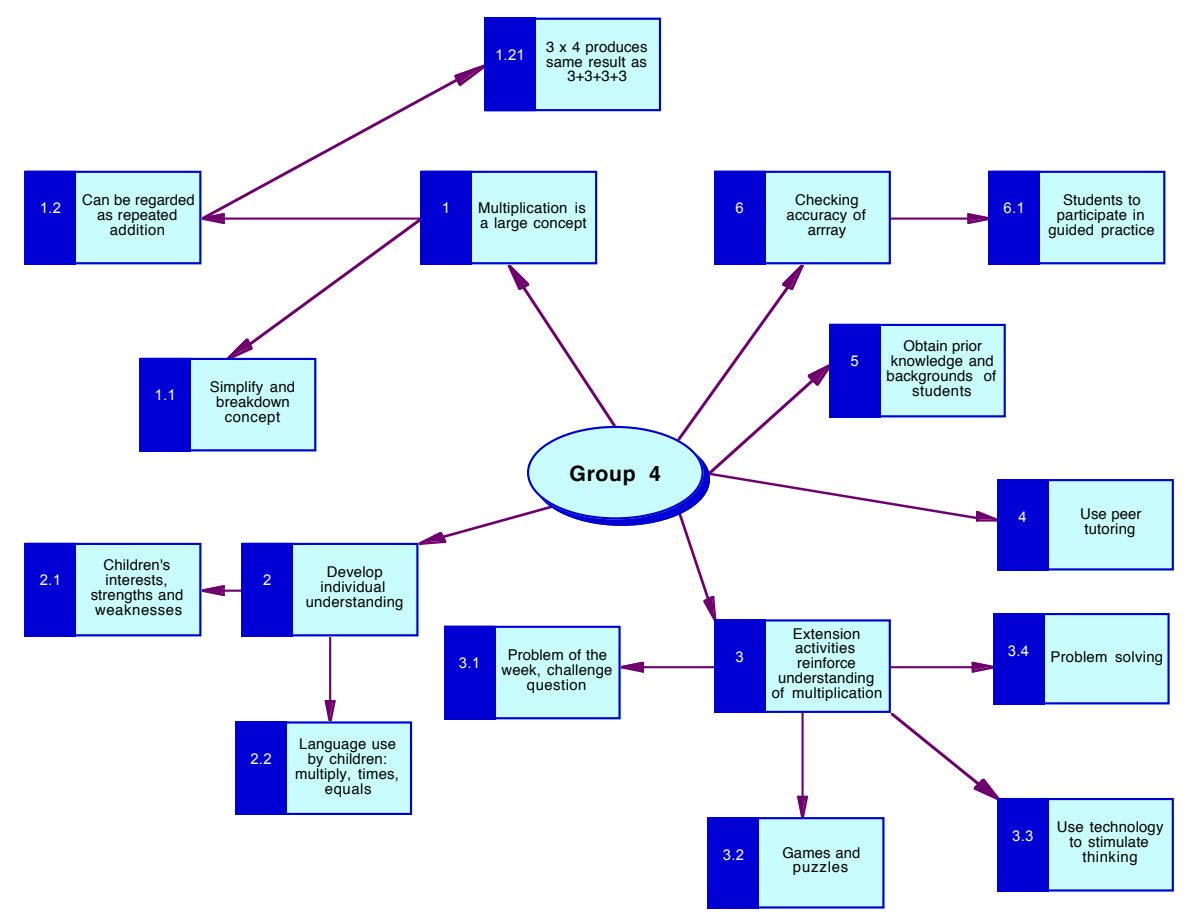

Figure 5: Concept map for Group 4 discussion

Overall, the interactions among the members in the four groups tended to cover the five major components of pedagogical content knowledge that were identified in the framework developed for the present study (Figure 1). Members in Group 1 engaged in a robust exchange of views that were related to the commutative property, and the modelling of this property with the use of arrays. Collectively, these exchanges demonstrate evidence of activation of knowledge related to three components of their pedagogical schema: representation, concept elaboration and patterns/ properties. The following responses from Group 1 draw attention to the need to go beyond pattern identification during teaching.

One of the biggest problems with the teaching of maths is that many teachers see patterns as things like bead patterns or simple colour patterns and do not recognise there place in other areas of mathematics like multiplication. 
If students are able to see the pattern in what they are doing, in this case it would be the teacher asking the children to express an array into a number sentence and using the kinds of materials to arrange the arrays to see relationships such as $6 \times 4=24$ and $4 \times 6=24$, will lead the students to a much deeper understanding and development of the concept.

In Group 4 (Node 3), there were considerations that addressed the issue of providing learning experiences that would help children investigate the concept of multiplication further. For example, it was suggested that problem solving could be a valuable strategy for independent exploration of multiplication. Views on this strategy also prompted other members to suggest the use of technology to foster thinking among children. These provide evidence of use of pedagogical knowledge related to design of learning activities and representation of the concept (Figure 1).

There were instances when the participants commented on the need to attend to children's individual differences and learning styles when teaching the concept of multiplication (Figure 3, Nodes 2 and 3; Figure 4, Node 4; Figure 5, Node 2). One exchange from Group 2 where participants exchanged views on the need to consider children's individual differences is presented below.

These activities would appeal to those students who have 'Interpersonal intelligence' through working in the groups and participating in discussion and 'Visual/Spatial' intelligence through studying and making the arrays.

Of course, there is also the 'Logical-Mathematical' intelligence incorporated through the content of the lesson. Even though I don't think it's possible to meet every learning style through every lesson, I do think this one would be better with the 'Bodily-Kinaesthetic' intelligence incorporated through using the students themselves in an array, or perhaps moving the desks into different arrays. This would excite those kids who like to be active and use their body for expression. How do you guys think that other learning styles could be met?

There was also evidence of accessing cognitive theories about the nature of learning. These theories were subsequently brought to bear on arguments about appropriate strategies for teaching multiplication. The WebCT environment also provided a convenient medium to pose questions and challenge other members to respond as demonstrated by the last sentence in the above exchange.

\section{Discussion}

This study explored components of the knowledge base of pre-service teachers that are relevant to multiplication and the teaching of multiplication. WebCT was used to facilitate online discussions among the 
participants in order to generate data about the above knowledge base. It was underpinned by the assumption that multiplication and multiplication situations are complex in nature and that appropriate modelling of this concept could be an effective strategy in helping young children acquire a meaningful understanding of the concept. It was hypothesised that student teachers with a greater repertoire of representations of multiplications and situations involving multiplications would activate this knowledge during the online discussions and prompt fellow students to engage them. The expectation was that WebCT postings would be exploited to depict multiplication in ways that would develop links between children's implicitly held understandings and the formal understandings that are stated in the goals of K-6 mathematics curriculum.

Student teachers' comments were analysed for evidence of understanding of multiplication concept and the teaching of the concept. This necessitated the construction of concept maps and the interpretation of the maps in terms of underlying knowledge schemas. The results of analysis of concept maps suggest that WebCT provided a convenient and non-threatening medium in which to generate descriptions about pre-service mathematics teachers' knowledge and understandings about multiplication. Overall, participants showed a high level of interest in exchanging not only their own views but also constructing robust arguments in support of or against positions taken by fellow student teachers through this medium.

Most of the participants exhibited a limited understanding of the subconcepts that are required for a deeper appreciation of multiplication by children. Clarkson (1998) reported similar results about shortcomings in beginning teachers' understanding of arithmetic concepts. While participants in the present study were knowledgeable about ways to use concrete material in the classroom, they were less forthcoming with rationale underlying the use of such resources. That is, participating teachers were unable to provide justifications for the 'cognitive scaffolding' that could be provided by the instructional materials that were suggested in the discussions. This pattern of results is interpreted as suggesting that the pre-service teachers have developed a limited repertoire of content and pedagogical content knowledge of multiplication.

One of the most commonly held views among the prospective teachers concerned repeated addition and place value of numbers that are involved in multiplication. However, they could have extended this in a number of ways to demonstrate properties of multiplication, particularly with the aid of Internet based resources. These results suggest that the student teachers in the present study were focused on showing that multiplication of two whole numbers could produce a third number that was larger than the 
initial two numbers. Repeated addition and its modelling indicated that student teachers viewed multiplication as a form of addition. This approach could provide children with an opportunity to 'see' the connection between numeration and the computational process that was considered to be pivotal in understanding numbers and operations (Hiebert \& Wearne, 1992; Vergnaud, 1988).

The strategy of modelling multiplication as repeated addition also addresses two key learning issues raised by Schwartz (1988) in relation to difficulties that could be experienced by young children when they shift from dealings with addition to multiplication. Unlike addition and subtraction, in multiplication situations children are expected to work with composite units as opposed to single units. Additionally, multiplication may involve either like or unlike quantities to produce a third quantity (the product). The latter attribute of the operation of multiplication, which is indicative of a deeper appreciation of the concept, was not borne out by the data. Nevertheless, the use of WebCT discussion in order to analyse modelling of multiplication as repeated addition appears to be an effective way to help the participating pre-service teachers reflect on the pedagogical demands of teaching this area of arithmetic.

Although two of the four groups explored the commutative property, none of their discussions examined the distributive and identity properties of multiplication, both of which are important in children's understanding of general relations and patterns that underlie multiplication. It would appear the student teachers did not regard these as an integral aspect of teaching multiplication.

A significant proportion of student teachers' exchanges focused on the array model of multiplication. The portrayal of multiplication in the array form would help children develop elementary notions of algebra, particularly the idea of modelling (National Council for Teachers of Mathematics, 2000). The arrangement of the numbers involved in a multiplicative operation in rows and columns provides an effective alternative representation of multiplication. The participants used array modelling correctly to demonstrate the commutative property. The exchanges also alluded to the need to relate the numbers that were involved in the operation with real life situations. This part of the discussion reveal an important component of pre-service teachers' pedagogical schema for multiplication, where they articulate the advantages of "concretising" the abstractions involved in understanding the operation. 
While there were important indicators of development of stores of schematised knowledge, one also observes the failure of prospective teachers in the present study to provide more varied and potentially richer learning experiences about multiplication. This could be attributed to a number of reasons. Firstly, the student teachers did not 'have' sufficient knowledge about multiplicative process. It is also possible that the lesson plan employed in the present study did not provide sufficient prompts to search a wider knowledge base than that revealed by the results reported here. Future studies need to examine this limitation and develop more sensitive focus questions.

The volume and richness of data that were generated by WebCT forums suggest that this medium not only permitted greater insight into a complex pedagogical issue in mathematics teaching, but also established a powerful learning forum. Further, as an ICT based medium WebCT facilitated the construction of new understandings among the participants about teaching a core concept in primary mathematics. Thus WebCT has inbuilt tools that not only facilitate the sharing of ideas, but also the activation of cognitions that help student teachers shift to new boundaries of understanding. The need to include this feature in the design of ICT based learning environments was advocated by Jonassen (1999).

A WebCT based learning forum can also be seen as an important venue to promote the growth of elaborate and sophisticated pedagogical content knowledge schemas among pre-service teachers. The results have significant implications for other pre-service teacher education courses and in service programs for mathematics teachers. It would seem that online discussions could be integrated into traditional forms of tertiary instruction as an effective strategy to help student teachers explore and reflect on their understandings about teaching concepts such as multiplication.

\section{References}

Chinnappan, M. (1998). Schemas and mental models in geometry problem solving. Educational Studies in Mathematics, 36(3), 201-217.

Clark, C. \& Peterson, P.L. (1986). Teachers' thought processes. In M. C. Wittrock (Ed.), Handbook of Research on Teaching (pp. 255-297). New York: Macmillan.

Clarkson, P. C. (1998). Beginning teachers' problems with fundamental mathematics. In C.Kanes, M.Goos \& E.Warren (Eds.), Teaching mathematics in new times (pp. 169-176). Brisbane, Australia: MERGA.

Cobb, P. (1994). Where is the mind? Constructivist and social perspectives on mathematical development. Educational Researcher, 23(7), 13-20. 
English, L.D. \& Halford, G.S. (1995). Mathematics education: Models and processes. Hillsdale, NJ: Lawrence Erlbaum.

Feiman-Nemser, S. (1990). Teacher preparation: Structural and conceptual alternatives. In W.R. Houston, M. Haberman \& J. Sikula (Eds.), Handbook of Research on Teacher Education (pp. 212-233). New York: Macmillan.

Hiebert, J. \& Wearne, D. (1992). Links between teaching and learning place value with understanding in first grade. Journal for Research in Mathematics Education, 23(2), 98-122.

Jonassen, D. H., Beissner, K. \& Yacci, M. A. (1993). Structural knowledge: Techniques for conveying, assessing, and acquiring structural knowledge, Hillsdale, NJ: Lawrence Erlbaum.

Jonassen, D. H. (1999). Designing constructivist learning environment. In C. M. Reigeluth (Ed), Instructional theories and models, (2nd ed, pp. 215-239). Mahwah, NJ: Lawrence Erlbaum.

Lawson, M.J. \& Chinnappan, M. (1994). Generative activity during geometry problem solving: Comparison of the performance of high-achieving and lowachieving students. Cognition and Instruction, 12(1), 61-93.

Leinhardt, G. (1987). The development of an expert explanation: An analysis of a sequence of subtraction lessons. Cognition and Instruction, 4(4), 225-282.

Marshall, S. P. (1995). Schemas in problem solving. NY: Cambridge University Press.

National Council of Teachers of Mathematics (2000). Curriculum and Evaluation Standards for School Mathematics. Reston, Va.: The Council.

Schoenfeld, A. H. (1992). Learning to think mathematically: Problem solving, metacognition, and sense making in mathematics. In D. A. Grows (Ed.), Handbook of research on mathematics teaching and learning (pp. 334-370). NY: Macmillan.

Schwartz, J. (1988). Intensive quantity and referent transforming arithmetic operations. In J.Hiebert and M.Behr (Eds.), Number concepts and operations in the middle grades (pp.41-52). Hillsdale, NJ: Lawrence Erlbaum.

Shulman, L.S. (1986). Paradigms and research programs in the study of teaching: A contemporary perspective. In M. C. Wittrock (Ed.) Handbook on Research in Teaching. (3rd ed., pp 3-36). New York: MacMillan.

Simon, M. A. (1995). Reconstructing mathematics pedagogy from a constructivist perspective. Journal for Research in Mathematics Education, 26, 114-254.

Vergnaud, G. (1988). Multiplicative structures. In J.Hiebert and M.Behr (Eds.), Number concepts and operations in the middle grades (pp. 141-161). Hillsdale, NJ: Lawrence Erlbaum.

Mohan Chinnappan $B S c$ (Maths), DipEd, BEd, MEd, $P h D$ is a Senior Lecturer in the University of Wollongong's Faculty of Education. Personal home page: http: / / edserver1.uow.edu.au:16080/ mohan/ 